\title{
Mental Health Among Parents of Children Aged $<18$ Years and Unpaid Caregivers of Adults During the COVID-19 Pandemic — United States, December 2020 and February-March 2021
}

\author{
Mark É. Czeisler ${ }^{1,2,3}$; Elizabeth A. Rohan, PhD, Stephanie Melillo, MPH ${ }^{4}$; Jennifer L. Matjasko, PhD ${ }^{4}$; Lara DePadilla, PhD ${ }^{4}$; Chirag G. Patel, DC ${ }^{4}$; \\ Matthew D. Weaver, PhD ${ }^{1,3,5}$; Alexandra Drane ${ }^{6}$; Sarah S. Winnay ${ }^{6}$; Emily R. Capodilupo ${ }^{6,7}$; Rebecca Robbins, PhD ${ }^{3,5}$; Joshua F. Wiley, PhD ${ }^{1}$; \\ Elise R. Facer-Childs, $\mathrm{PhD}^{1}$; Laura K. Barger, PhD ${ }^{1,3,5}$; Charles A. Czeisler, PhD, MD ${ }^{1,3,5}$; Mark E. Howard, PhD ${ }^{1,2,8 *}$; Shantha M.W. Rajaratnam, PhD ${ }^{1,2,3,5 *}$
}

Early during the COVID-19 pandemic, nearly two thirds of unpaid caregivers of adults reported adverse mental or behavioral health symptoms, compared with approximately one third of noncaregivers ${ }^{\dagger}(1)$. In addition, $27 \%$ of parents of children aged $<18$ years reported that their mental health had worsened during the pandemic (2). To examine mental health during the COVID-19 pandemic among U.S. adults on the basis of their classification as having a parenting role (i.e., unpaid persons caring for children and adolescents aged $<18$ years, referred to as children in this report) or being an unpaid caregiver of adults (i.e., persons caring for adults aged $\geq 18$ years), ${ }^{\$} \mathrm{CDC}$ analyzed data from cross-sectional surveys that were administered during December 2020 and FebruaryMarch 2021 for The COVID-19 Outbreak Public Evaluation (COPE) Initiative. Respondents were categorized as parents only, caregivers of adults only, parents-caregivers (persons in both roles), or nonparents/noncaregivers (persons in neither role). Adjusted odds ratios (aORs) for any adverse mental health symptoms, particularly suicidal ideation, were higher among all respondents who were parents, caregivers of adults, or both compared with respondents who were nonparents/ noncaregivers and were highest among persons in both roles

\footnotetext{
*These authors contributed equally to this report.

${ }^{\dagger} \mathrm{https} / /$ www.medrxiv.org/content/10.1101/2021.02.02.21251042v1

$\$$ Parents and unpaid caregivers of adults were self-identified. Parents were defined as persons who had provided unpaid care to relatives or friends aged $<18$ years to help them take care of themselves at any time during the last 3 months. Unpaid caregivers of adults were defined as persons who had provided unpaid care to relatives or friends aged $\geq 18$ years to help them take care of themselves at any time during the last 3 months. Respondents answered questions about these two roles separately. Respondents were categorized as parents only, caregivers of adults only, parents-caregivers (persons in both roles), or nonparents/noncaregivers. Whether adults who reported they were in parenting roles were biologic or legal parents or guardians of the children for whom they were providing care is not known, nor is it known whether adults were legal dependents of their caregivers.

The COPE Initiative (https://www.thecopeinitiative.org/) is designed to assess public attitudes, behaviors, and beliefs related to the COVID-19 pandemic and to evaluate the mental and physical health consequences of the pandemic. The COPE Initiative surveys included in this analysis were administered by Qualtrics, LLC (https://www.qualtrics.com), a commercial survey company with a network of participant pools comprising hundreds of suppliers and with varying recruitment methodologies that include digital advertisements and promotions, word-of-mouth and membership referrals, social networks, television and radio advertisements, and offline mail-based approaches.
}

(parents-caregivers) (any adverse mental health symptoms: $\mathrm{aOR}=5.1,95 \%$ confidence interval $[\mathrm{CI}]=4.1-6.2$; serious suicidal ideation: $\mathrm{aOR}=8.2,95 \% \mathrm{CI}=6.5-10.4)$. These findings highlight that parents and caregivers, especially those balancing roles both as parents and caregivers, experienced higher levels of adverse mental health symptoms during the COVID-19 pandemic than adults without these responsibilities. Caregivers who had someone to rely on for support had lower odds of experiencing any adverse mental health symptoms. Additional measures are needed to improve mental health among parents, caregivers, and parents-caregivers.

Among 16,384 eligible and invited unique respondents, ${ }^{* *}$ 10,469 (63.9\%) completed English-language, Internet-based surveys administered to Qualtrics panels for The COPE Initiative during distinct intervals (December 6-27, 2020, and February 16-March 8, 2021). The nonprobability demographic quota sample was weighted to closely align with the distribution of the U.S. population by sex, age, and race/ ethnicity. ${ }^{\dagger \dagger}$ Data for explanatory and outcome variables were obtained from 10,444 (99.8\%) respondents. Respondents described their parenting and caregiving roles, completed screening instruments for symptoms of anxiety and depression ${ }^{\$ \$}$ and COVID-19 trauma- and stressor-related disorders

\footnotetext{
** Eligibility to complete surveys was determined after electronic contact with potential participants who met criteria of age $\geq 18$ years and U.S. residence. Age and residence were assessed using screening questions without indication of eligibility criteria before survey commencement. Country-specific geolocation verification via IP address mapping was used to ensure respondents were in the United States. Qualtrics, LLC, conducted data quality screening including algorithmic and keystroke analysis for attention patterns, clickthrough behavior, duplicate responses, machine responses, and inattentiveness.

$\dagger \dagger$ Additional information on quota sampling, a nonprobabilistic sampling method, is available at https://www.qualtrics.com/experience-management/ research/sampling-methods/. Demographic quotas were set for sex, age, race, and ethnicity using questions and national U.S. adult population estimates from the 2019 American Community Survey. After the surveys were conducted, iterative proportional fitting and weight trimming were applied to the overall sample to match 2019 American Community Survey estimates for sex, age, and combined race/ethnicity. Survey weighting was performed using the R survey package (version 3.29; R Foundation).

$\$ \$$ Symptoms of anxiety and depression were assessed via the four-item Patient Health Questionnaire (PHQ-4), which refer to anxiety and depression symptoms experienced over the past 2 weeks. Those who scored $\geq 3$ out of 6 on the Generalized Anxiety Disorder (GAD-2) or Patient Health Questionnaire (PHQ-2) subscales were considered symptomatic for these respective conditions.
} 
(TSRDs), 99 and reported whether during the past 30 days they had wished they were dead or could go to sleep and not wake up (passive suicidal ideation) or had seriously considered trying to kill themselves (serious suicidal ideation). ${ }^{* * *}$

Respondents were grouped based on their roles as 1) only parents of children aged $<18$ years (parents only), 2) only caregivers of adults aged $\geq 18$ years (caregivers only), 3 ) having both roles (parents-caregivers), or 4) having neither role (nonparents/noncaregivers). Multivariable weighted logistic regression was used to estimate aORs by group for symptoms of anxiety or depression or COVID-19TSRDs, passive suicidal ideation, serious suicidal ideation, or any of these symptoms. Covariates included gender, age, race/ethnicity, sexual orientation, disability status, ${ }^{\dagger \dagger}$ education, U.S. Census region ${ }^{\$ \$ \$}$ and urbanicity $\mathbf{9 9}$ of residence, employment characteristics, ${ }^{* * * *}$ and survey wave. Models also estimated aORs for adverse mental health symptoms by the following reasons for providing care for adults: 1) age-related health decline, 2) cognitive impairments (e.g., Alzheimer disease), 3) chronic medical conditions (e.g., cancer), 4) acute medical conditions (e.g., recovery after surgery), 5) mental health or substance use conditions, 6) active COVID-19 illness, 7) risk for severe COVID-19-associated

99 Disorders classified as TSRDs in the Diagnostic and Statistical Manual of Mental Disorders, Fifth Edition, include posttraumatic stress disorder (PTSD), acute stress disorder (ASD), and adjustment disorders, among others. Symptoms of a TSRD attributed to the COVID-19 pandemic were assessed via the six-item Impact of Event scale (IES-6) to screen for overlapping symptoms of PTSD, ASD, and adjustment disorders. The COVID-19 pandemic was specified as the traumatic exposure to record peritraumatic and posttraumatic symptoms associated with the range of stressors introduced by the COVID-19 pandemic. Those who scored $\geq 1.75$ out of 4 were considered symptomatic.

*** For questions related to suicidal ideation, participants were informed that responses were deidentified and that direct support could not be provided to those who reported substance use behavior or suicidal ideation. Regarding suicidal ideation, all respondents were provided the following: "This survey is anonymous, so we cannot provide direct support. If you would like crisis support, please contact the National Suicide Prevention Lifeline, 1-800-273-TALK (8255, or chat line) for help for yourself or for others." Passive suicidal ideation was assessed using an item from the ColumbiaSuicide Severity Rating Scale adapted to refer to the past 30 days: "At any time in the past 30 days, have you wished you were dead or wished you could go to sleep and not wake up?" Serious suicidal ideation was assessed using an item from the National Survey on Drug Use and Health adapted to refer to the past 30 days: "At any time in the past 30 days, did you seriously think about trying to kill yourself?"

$\nmid \dagger \dagger$ Persons who had a disability were defined as such based on a qualifying response to either one of the two following questions: "Are you limited in any way in any activities because of physical, mental, or emotional condition?" and "Do you have any health conditions that require you to use special equipment, such as a cane, wheelchair, special bed, or special telephone?" https://www.cdc.gov/brfss/questionnaires/pdf-ques/2015-brfssquestionnaire-12-29-14.pdf

$\$ \$ \$ \$$ https://www2.census.gov/geo/pdfs/maps-data/maps/reference/us_regdiv.pdf

999 https://www.hrsa.gov/rural-health/about-us/definition/datafiles.html

**** Employment characteristics included employment status (employed, unemployed, retired, or student only), weekly paid work hours, and percentage of work hours completed remotely versus on-site. illness, or 8) other. Additional models among all caregivers of adults, which also adjusted for parenting, duration of caregiving, hours of caregiving per week, and person receiving care, were used to estimate aORs by level of agreement with statements about caregiving-related financial strain, family strife, preparedness, support, confidence, personal freedom, positive feelings, and resentment. ${ }^{\dagger \dagger \dagger}$ Variance inflation factors for all variables with aOR estimates were less than six, indicating acceptable multicollinearity. $\$ \$ \$ \$$

Participants provided informed electronic consent. Twosided $\mathrm{p}$ values $<0.05$ were considered statistically significant. Rounded, weighted values are reported. Analyses were conducted using Python (version 3.7.8; Python Software Foundation) and R (version 4.0.2; R Foundation) using the R survey package (version 3.29; R Foundation). The Monash University Human Research Ethics Committee reviewed and approved the study. This activity was also reviewed by CDC and was conducted consistent with applicable federal law and CDC policy. 9999

Overall, $42.5 \%$ of the 10,444 U.S. adult respondents identified as parents of children, caregivers of adults, or both, including $8.4 \%$ as parents only, $11.2 \%$ as caregivers only, and $22.9 \%$ as parents-caregivers (Table 1). Among all respondents who were parents, caregivers, or parents-caregivers, $45.0 \%$ were women and $50.2 \%$ were aged $25-44$ years. The distribution by race/ethnicity was similar to those of the overall sample and the U.S. adult population. A total of $71.4 \%$ of parents or caregivers reported paid employment in addition to their parenting or unpaid caregiving roles.

Approximately $70 \%$ of all caregivers (parents only, caregivers of adults only, or those with both roles) reported adverse mental health symptoms, including symptoms of anxiety or depression (55.3\%), COVID-19 TSRDs (53.8\%), or passive $(39.3 \%)$ or serious $(32.2 \%)$ suicidal ideation (Table 2$)$. Among 2,391 parents-caregivers, approximately $85 \%$ experienced one or more adverse mental health symptoms, and approximately $50 \%$ reported past-month serious suicidal ideation. Parenting and caregiving were significantly positively associated with

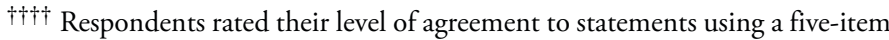
Likert scale $(1=$ strongly disagree; $2=$ disagree; $3=$ neither agree nor disagree; 4 = agree; 5 = strongly agree) taken from the ARCHANGELS short-form Caregiver Intensity Index (CII), a copyrighted instrument available for use only with permission. Responses were trichotomized to disagree ( 1 and 2 ), neutral (3), or agree ( 4 and 5). CII was administered to all unpaid caregivers of adults.

$\$ S \$ S$ The maximum acceptable level of variance inflation factor cutoff was set at 10 , which signals high multicollinearity (i.e., when two or more explanatory variables in a multivariable model are highly correlated). https:// www.itl.nist.gov/div898/software/dataplot/refman2/auxillar/vif.htm

999945 C.F.R. part 46, 21 C.F.R. part 56; 42 U.S.C. Sect. 241(d); 5 U.S.C. Sect. 552a; 44 U.S.C. Sect. 3501 et seq. 
TABLE 1. Demographic characteristics of respondents, by parent/caregiver role - The COVID-19 Outbreak Public Evaluation Initiative, United States, December 2020 and February-March 2021

\begin{tabular}{|c|c|c|c|c|c|c|}
\hline \multirow[b]{2}{*}{ Characteristic } & \multicolumn{6}{|c|}{ Weighted no. (\%)* } \\
\hline & Total & $\begin{array}{l}\text { Nonparents/ } \\
\text { Noncaregivers }\end{array}$ & $\begin{array}{l}\text { Parents only, caregivers of } \\
\text { adults only, and } \\
\text { parents-caregivers }\end{array}$ & $\begin{array}{c}\text { Parents } \\
\text { only }\end{array}$ & $\begin{array}{l}\text { Caregivers of } \\
\text { adults only }\end{array}$ & $\begin{array}{l}\text { Parents- } \\
\text { caregivers }\end{array}$ \\
\hline Total & $10,444(100)$ & $6,008(57.5)$ & $4,436(42.5)$ & $875(8.4)$ & $1,170(11.2)$ & $2,391(22.9)$ \\
\hline \multicolumn{7}{|l|}{ Gender $§$} \\
\hline Female & $5,138(49.2)$ & $3,144(52.3)$ & $1,995(45.0)$ & $510(58.2)$ & $611(52.2)$ & 874 (36.6) \\
\hline Male & $5,227(50.1)$ & $2,827(47.1)$ & $2,400(54.1)$ & $360(41.2)$ & $552(47.2)$ & $1,487(62.2)$ \\
\hline Transgender & $58(0.6)$ & $26(0.4)$ & $32(0.7)$ & - & - & $26(1.1)$ \\
\hline \multicolumn{7}{|l|}{ Age group, yrs } \\
\hline $18-24$ & $1,248(11.9)$ & $549(9.1)$ & $699(15.7)$ & $91(10.5)$ & $145(12.4)$ & $462(19.3)$ \\
\hline $25-44$ & $3,605(34.5)$ & $1,377(22.9)$ & $2,227(50.2)$ & $426(48.6)$ & $393(33.6)$ & $1,409(58.9)$ \\
\hline $45-64$ & $3,419(32.7)$ & $2,293(38.2)$ & $1,126(25.4)$ & $266(30.4)$ & $427(36.5)$ & $433(18.1)$ \\
\hline$\geq 65$ & $2,172(20.8)$ & $1,789(29.8)$ & $384(8.6)$ & $92(10.5)$ & $205(17.5)$ & $87(3.7)$ \\
\hline \multicolumn{7}{|l|}{ Race/Ethnicity } \\
\hline White, non-Hispanic & $6,297(60.3)$ & $3,660(60.9)$ & $2,637(59.5)$ & $550(62.9)$ & $711(60.7)$ & $1,376(57.6)$ \\
\hline Black, non-Hispanic & $1,297(12.4)$ & $766(12.7)$ & $531(12.0)$ & $103(11.8)$ & $135(11.5)$ & $293(12.2)$ \\
\hline Asian, non-Hispanic & $589(5.6)$ & $408(6.8)$ & $181(4.1)$ & $43(4.9)$ & $67(5.7)$ & $71(3.0)$ \\
\hline Other, multiple races, non-Hispanic? & $382(3.7)$ & $220(3.7)$ & $162(3.6)$ & $36(4.2)$ & $61(5.2)$ & $64(2.7)$ \\
\hline Hispanic, any race & $1,880(18.0)$ & 955 (15.9) & $925(20.9)$ & $142(16.3)$ & $196(16.8)$ & $587(24.5)$ \\
\hline \multicolumn{7}{|l|}{ Employment status } \\
\hline Employed & $5813(55.7)$ & $2,645(44.0)$ & $3,167(71.4)$ & $518(59.2)$ & $654(55.9)$ & $1,995(83.4)$ \\
\hline$\leq 40 \mathrm{hrs},<20 \%$ remote & $1,500(14.4)$ & $970(16.1)$ & $531(12.0)$ & $153(17.5)$ & $179(15.3)$ & $199(8.3)$ \\
\hline$\leq 40 \mathrm{hrs}, 20 \%-80 \%$ remote & $1,209(11.6)$ & $448(7.5)$ & $761(17.2)$ & $102(11.7)$ & $144(12.3)$ & $515(21.5)$ \\
\hline$\leq 40 \mathrm{hrs},>80 \%$ remote & $877(8.4)$ & $490(8.2)$ & $387(8.7)$ & $76(8.7)$ & $82(7.0)$ & $228(9.5)$ \\
\hline$>40 \mathrm{hrs},<20 \%$ remote & $568(5.4)$ & $341(5.7)$ & $227(5.1)$ & $66(7.6)$ & $79(6.8)$ & $81(3.4)$ \\
\hline$>40 \mathrm{hrs}, 20 \%-80 \%$ remote & $1,120(10.7)$ & $224(3.7)$ & $896(20.2)$ & $80(9.1)$ & $117(10.0)$ & $699(29.2)$ \\
\hline$>40 \mathrm{hrs},>80 \%$ remote & $539(5.2)$ & $172(2.9)$ & $366(8.3)$ & $41(4.7)$ & $53(4.6)$ & $272(11.4)$ \\
\hline Unemployed & $1,791(17.2)$ & $1,160(19.3)$ & $632(14.2)$ & $208(23.8)$ & $215(18.4)$ & $208(8.7)$ \\
\hline Retired & $2,517(24.1)$ & $2,010(33.5)$ & $508(11.4)$ & $124(14.2)$ & $265(22.7)$ & $119(5.0)$ \\
\hline Student & $322(3.1)$ & $193(3.2)$ & $129(2.9)$ & $24(2.8)$ & $36(3.0)$ & $69(2.9)$ \\
\hline \multicolumn{7}{|l|}{ Duration in parenting/caregiving role } \\
\hline$<3 \mathrm{mos}$ & - & - & $993(22.4)$ & $183(20.9)$ & $357(30.5)$ & $454(19.0)$ \\
\hline $4-12$ mos & - & - & $1,368(30.8)$ & $180(20.5)$ & $264(22.6)$ & $924(38.6)$ \\
\hline$>1 \mathrm{yr}$ & - & - & $2,075(46.8)$ & $513(58.6)$ & $549(46.9)$ & $1,013(42.4)$ \\
\hline \multicolumn{7}{|l|}{ Parenting, hrs/wk } \\
\hline$<10$ & - & - & - & $145(16.5)$ & - & $261(10.9)$ \\
\hline $10-20$ & - & - & - & $207(23.7)$ & - & $377(15.8)$ \\
\hline $21-40$ & - & - & - & $211(24.1)$ & - & $570(23.8)$ \\
\hline $41-60$ & - & - & - & $92(10.5)$ & - & $374(15.7)$ \\
\hline$>60$ & - & - & - & $220(25.2)$ & - & $808(33.8)$ \\
\hline \multicolumn{7}{|l|}{ Adult caregiving, hrs/wk } \\
\hline$<10$ & - & - & - & - & $317(27.1)$ & $239(10.0)$ \\
\hline $10-20$ & - & - & - & - & $363(31.0)$ & $457(19.1)$ \\
\hline $21-40$ & - & - & - & - & $229(19.6)$ & $606(25.4)$ \\
\hline $41-60$ & - & - & - & - & $80(6.8)$ & $352(14.7)$ \\
\hline$>60$ & - & - & - & - & $182(15.6)$ & $737(30.8)$ \\
\hline \multicolumn{7}{|l|}{ Reason for providing care for adults** } \\
\hline Age-related health decline & - & - & - & - & $477(40.8)$ & $587(24.5)$ \\
\hline Cognitive impairments & - & - & - & - & $188(16.1)$ & $339(14.2)$ \\
\hline Chronic health condition & - & - & - & - & $303(25.9)$ & $662(27.7)$ \\
\hline Acute health condition & - & - & - & - & $118(10.1)$ & 405 (16.9) \\
\hline Mental health or substance use condition & - & - & - & - & $162(13.9)$ & $573(24.0)$ \\
\hline Active case of COVID-19 & - & - & - & - & $96(8.2)$ & $659(27.5)$ \\
\hline Risk for severe COVID-19 & - & - & - & - & $190(16.3)$ & $637(26.6)$ \\
\hline Other & - & - & - & - & $165(14.1)$ & $155(6.5)$ \\
\hline
\end{tabular}

See table footnotes on the next page. 
TABLE 1. (Continued) Demographic characteristics of respondents, by parent/caregiver role - The COVID-19 Outbreak Public Evaluation Initiative, United States, December 2020 and February-March 2021

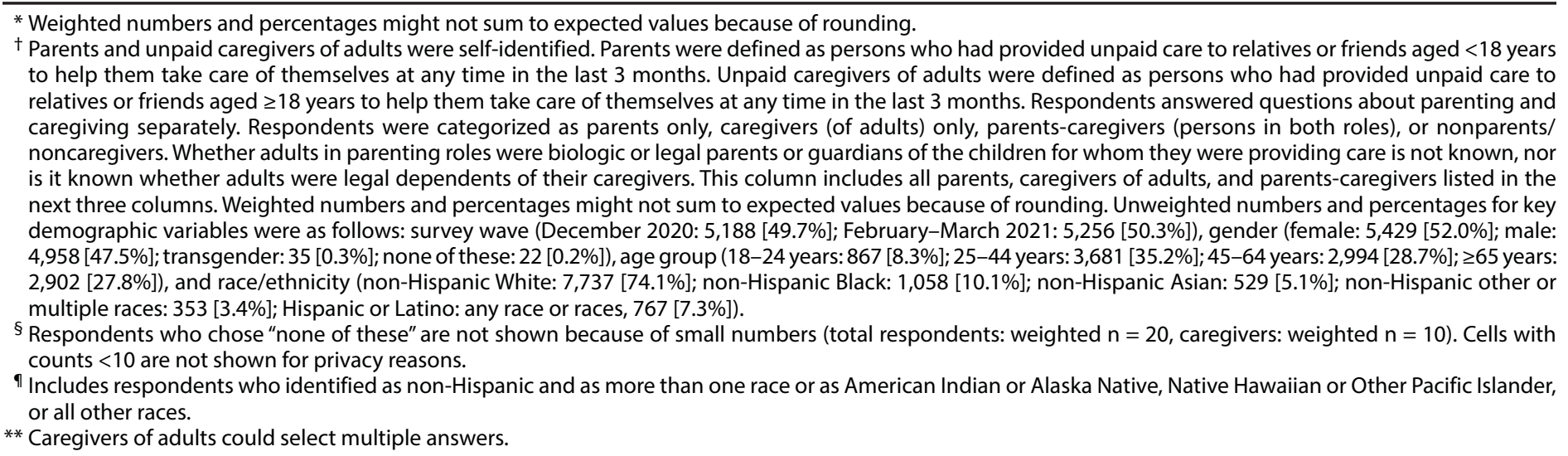

each adverse mental health symptom compared with being a nonparent/noncaregiver (for one or more symptoms, parents only: $\mathrm{aOR}=1.5$; caregivers only: $\mathrm{aOR}=1.8$; parents-caregivers: $\mathrm{aOR}=5.1$ ) and was particularly high for serious suicidal ideation among parents-caregivers compared with nonparents/ noncaregivers $(\mathrm{aOR}=8.2)$. Among respondents providing care for an adult for a given health condition compared with those not providing care for that condition, the highest aORs for adverse mental health symptoms were observed for caregivers of adults with mental health or substance use conditions (e.g., one or more symptoms: $\mathrm{aOR}=5.0$ ), adults with an active case of COVID-19 (aOR = 4.4), or adults at risk for severe COVID-19 $(\mathrm{aOR}=3.9)$ (Table 2). Higher aORs for all adverse mental health symptoms were also observed for caregivers who were caring for adults with acute health conditions, chronic health conditions, cognitive impairments, and age-related health decline.

Among all caregivers of adults (adult caregivers only and parents-caregivers), those who agreed with the statements that they had experienced caregiving-related family disagreements or resented their caregiving responsibilities had approximately three times the odds for any adverse mental health symptoms (Figure) compared with those who disagreed with these statements. Similarly, aORs for any adverse mental health symptoms were approximately twice as high for caregivers who agreed that they felt underprepared as a caregiver, did not have the personal freedom they desired, or had to decrease living expenses to help pay for things, compared with caregivers who did not agree with these statements. Conversely, persons who had someone to rely on for support had lower odds of experiencing any adverse mental health symptoms.

\section{Discussion}

Approximately $40 \%$ of U.S. adults surveyed in late 2020 or early 2021 reported having parenting responsibilities, adult caregiving responsibilities, or both. Overall, 70\% of all caregivers (parents only, caregivers of adults only, or those with both roles) reported recent adverse mental health symptoms, including symptoms of anxiety or depression, COVID-19 TSRDs, or suicidal ideation. Of particular concern, 85\% of respondents with both parenting responsibilities and adult caregiving responsibilities experienced adverse mental health symptoms, and approximately 50\% reported past-month serious suicidal ideation, with eight times the odds of serious suicidal ideation compared with nonparents/noncaregivers.

Caregivers of adults with mental health or substance use conditions, adults currently ill with COVID-19, or adults at risk for severe COVID-19 reported more adverse mental health symptoms than did caregivers of adults with other conditions, highlighting the need for education and support for caregivers in these roles. Social factors, such as financial strain, feeling a lack of preparedness for or resentment of caregiving, a lack of freedom, and family conflict were also associated with adverse mental health. The lower odds of having any adverse mental health symptoms based on the perception of having a person to rely on for support is encouraging. Because employment and caregiving responsibilities might limit the time available to seek help, telehealth and Internet-based interventions (3) might improve caregiver mental health; however, Internet access might be limited for some populations, particularly those with lower incomes. In addition, adult day services centers might benefit the mental health of caregivers and of those for whom they are providing care (4). ${ }^{* * * * *}$ Finally,

\footnotetext{
***** https://www.cdc.gov/coronavirus/2019-ncov/community/adult-day-careservice-centers.html
} 
TABLE 2. Prevalence of and adjusted odds ratios for adverse mental health symptoms, by parent/caregiver role and reason for providing care for adults - The COVID-19 Outbreak Public Evaluation Initiative, United States, December 2020 and February-March 2021

\begin{tabular}{|c|c|c|c|c|c|c|c|c|c|c|c|c|}
\hline \multirow{3}{*}{$\begin{array}{l}\begin{array}{l}\text { Caregiver role } \\
\text { and reason for } \\
\text { providing care }\end{array} \\
\begin{array}{l}\text { Total, } \\
\text { no. }(\%) \\
(95 \% \mathrm{Cl})^{4}\end{array}\end{array}$} & \multicolumn{12}{|c|}{ Symptoms } \\
\hline & \multicolumn{2}{|c|}{ Total } & \multicolumn{2}{|c|}{$\begin{array}{c}\text { Anxiety or } \\
\text { depression* }\end{array}$} & \multicolumn{2}{|c|}{ COVID-19 TSRD ${ }^{\dagger}$} & \multicolumn{2}{|c|}{$\begin{array}{l}\text { Past-month passive } \\
\text { suicidal ideation }^{\S}\end{array}$} & \multicolumn{2}{|c|}{$\begin{array}{l}\text { Past-month serious } \\
\text { suicidal ideation }^{\S}\end{array}$} & \multicolumn{2}{|c|}{$\begin{array}{l}\text { Any of these } \\
\text { symptoms }\end{array}$} \\
\hline & $\begin{array}{r}10,444 \\
(100)\end{array}$ & - & $\begin{array}{l}3,780 \\
(36.2)\end{array}$ & $(35.1-37.3)$ & $\begin{array}{l}3,596 \\
(34.4)\end{array}$ & $(33.3-35.5)$ & $\begin{array}{l}2,321 \\
(22.2)\end{array}$ & $(21.2-23.2)$ & $\begin{array}{l}1,697 \\
(16.2)\end{array}$ & (15.4-17.1) & $\begin{array}{l}5,001 \\
(47.9)\end{array}$ & $(46.8-49.0)$ \\
\hline \multicolumn{13}{|c|}{ Parent role/caregiver role, ${ }^{* *}$ no. $(\%)(95 \% \mathrm{Cl})^{\uparrow}$} \\
\hline $\begin{array}{l}\text { Nonparent/ } \\
\text { Noncaregiver }\end{array}$ & $\begin{array}{l}6,008 \\
(57.5)\end{array}$ & $(56.4-58.7)$ & $\begin{array}{l}1,327 \\
(22.1)\end{array}$ & $(20.8-23.4)$ & $\begin{array}{l}1,209 \\
(20.1)\end{array}$ & $(18.8-21.4)$ & $\begin{array}{r}580 \\
(9.6)\end{array}$ & $(8.7-10.7)$ & $\begin{array}{r}269 \\
(4.5)\end{array}$ & $(3.8-5.3)$ & $\begin{array}{l}1,925 \\
(32.0)\end{array}$ & $(30.6-33.5)$ \\
\hline $\begin{array}{l}\text { Parent only, caregiver } \\
\text { of adults only, or } \\
\text { parent-caregiver }\end{array}$ & $\begin{array}{l}4,436 \\
(42.5)\end{array}$ & $(41.3-43.6)$ & $\begin{array}{l}2,453 \\
(55.3)\end{array}$ & $(53.4-57.2)$ & $\begin{array}{l}2,387 \\
(53.8)\end{array}$ & $(51.9-55.7)$ & $\begin{array}{l}1,741 \\
(39.3)\end{array}$ & $(37.4-41.1)$ & $\begin{array}{l}1,428 \\
(32.2)\end{array}$ & $(30.4-34.0)$ & $\begin{array}{l}3,076 \\
(69.3)\end{array}$ & $(67.6-71.0)$ \\
\hline Parent only & $\begin{array}{r}875 \\
(8.4)\end{array}$ & $(7.8-9.0)$ & $\begin{array}{r}315 \\
(35.9)\end{array}$ & $(32.2-39.8)$ & $\begin{array}{r}304 \\
(34.8)\end{array}$ & $(31.0-38.7)$ & $\begin{array}{r}162 \\
(18.5)\end{array}$ & $(15.2-22.2)$ & $79(9.0)$ & $(6.9-11.4)$ & $\begin{array}{r}443 \\
(50.6)\end{array}$ & $(46.6-54.6)$ \\
\hline $\begin{array}{l}\text { Caregiver of adults } \\
\text { only }\end{array}$ & $\begin{array}{l}1,170 \\
(11.2)\end{array}$ & $(10.4-12.0)$ & $\begin{array}{r}454 \\
(38.8)\end{array}$ & $(35.2-42.5)$ & $\begin{array}{r}425 \\
(36.3)\end{array}$ & $(32.8-40.0)$ & $\begin{array}{r}187 \\
(16.0)\end{array}$ & $(13.5-18.7)$ & $\begin{array}{r}118 \\
(10.1)\end{array}$ & $(8.1-12.4)$ & $\begin{array}{r}591 \\
(50.5)\end{array}$ & $(46.8-54.2)$ \\
\hline Parent-caregiver & $\begin{array}{l}2,391 \\
(22.9)\end{array}$ & $(21.9-23.9)$ & $\begin{array}{l}1,685 \\
(70.5)\end{array}$ & $(67.9-72.9)$ & $\begin{array}{l}1,658 \\
(69.3)\end{array}$ & $(66.8-71.8)$ & $\begin{array}{l}1,392 \\
(58.2)\end{array}$ & $(55.6-60.9)$ & $\begin{array}{l}1,232 \\
(51.5)\end{array}$ & $(48.8-54.2)$ & $\begin{array}{l}2,043 \\
(85.4)\end{array}$ & $(83.5-87.2)$ \\
\hline \multicolumn{13}{|c|}{ Parent role/caregiver role, ${ }^{* *}$ aOR $(95 \% \mathrm{Cl})^{\dagger \dagger}$} \\
\hline Parent only & - & - & & $1.4(1.1-1.7)$ & & $1.5(1.2-1.9)$ & & $1.5(1.2-2.0)$ & & $1.6(1.1-2.2)$ & & $1.5(1.2-1.8)$ \\
\hline Adult caregiver only & - & - & & $1.9(1.6-2.3)$ & & $1.8(1.5-2.2)$ & & $1.3(1.0-1.7)$ & & $1.7(1.2-2.3)$ & & $1.8(1.5-2.1)$ \\
\hline Parent-caregiver & - & - & & $3.7(3.1-4.5)$ & & $3.6(3.1-4.3)$ & & $5.8(4.8-7.1)$ & & $8.2(6.5-10.4)$ & & $5.1(4.1-6.2)$ \\
\hline \multicolumn{13}{|c|}{ Reason for care for adults, aOR $(95 \% \mathrm{Cl})^{\S \S}$} \\
\hline $\begin{array}{l}\text { Age-related health } \\
\text { decline }\end{array}$ & - & - & & $1.8(1.4-2.3)$ & & $1.8(1.5-2.3)$ & & $1.4(1.1-1.8)$ & & $1.8(1.3-2.3)$ & & $1.9(1.5-2.4)$ \\
\hline Cognitive challenges & - & - & & $2.0(1.5-2.7)$ & & $2.0(1.5-2.6)$ & & $2.3(1.7-3.0)$ & & $3.1(2.2-4.4)$ & & $2.2(1.7-2.8)$ \\
\hline $\begin{array}{l}\text { Chronic health } \\
\text { condition }\end{array}$ & - & - & & $2.8(2.2-3.5)$ & & $1.9(1.6-2.4)$ & & $2.8(2.2-3.5)$ & & $3.3(2.5-4.3)$ & & $2.3(1.8-2.9)$ \\
\hline Acute health condition & - & - & & $2.7(1.9-3.7)$ & & $2.8(2.1-3.7)$ & & $2.4(1.8-3.2)$ & & $3.6(2.6-4.9)$ & & $3.0(2.1-4.3)$ \\
\hline $\begin{array}{l}\text { Mental health or } \\
\text { substance use } \\
\text { condition }\end{array}$ & - & - & & $3.7(2.8-5.0)$ & & $3.7(2.8-4.8)$ & & $3.0(2.3-3.9)$ & & $3.8(2.9-5.0)$ & & $5.0(3.7-6.9)$ \\
\hline $\begin{array}{l}\text { Active case of } \\
\text { COVID-19 }\end{array}$ & - & - & & $3.8(2.7-5.1)$ & & $3.1(2.3-4.1)$ & & $4.2(3.2-5.6)$ & & $5.5(4.1-7.5)$ & & $4.4(3.0-6.4)$ \\
\hline $\begin{array}{l}\text { Risk for severe } \\
\text { COVID-19 }\end{array}$ & - & - & & $3.4(2.6-4.4)$ & & $2.8(2.2-3.6)$ & & $3.5(2.8-4.5)$ & & $4.7(3.6-6.1)$ & & $3.9(3.0-5.2)$ \\
\hline Other & - & - & & $1.6(1.1-2.5)$ & & $1.4(1.0-1.9)$ & & $1.2(0.8-1.9)$ & & $2.4(1.6-3.6)$ & & $1.5(1.0-2.2)$ \\
\hline
\end{tabular}

Abbreviations: $\mathrm{aOR}=$ adjusted odds ratio; $\mathrm{ASD}=$ acute stress disorder; $\mathrm{Cl}=$ confidence interval; $\mathrm{Cll}=$ Caregiving Intensity Index; GAD-2 = two-item Generalized Anxiety Disorder scale; IES-6 = six-item Impact of Event scale; PHQ-2 = two-item Patient Health Questionnaire; PHQ-4 = four-item Patient Health Questionnaire; PTSD = posttraumatic stress disorder; TSRD = trauma- and stressor-related disorder.

* Symptoms of anxiety and depression were assessed via PHQ-4. Those who scored $\geq 3$ out of 6 on the GAD-2 or PHQ-2 subscales were considered symptomatic for anxiety or depression symptoms.

† Disorders classified as TSRDs in the Diagnostic and Statistical Manual of Mental Disorders, Fifth Edition, include PTSD, ASD, and adjustment disorders, among others. Symptoms of a TSRD attributed to the COVID-19 pandemic were assessed via IES-6 to screen for overlapping symptoms of PTSD, ASD, and adjustment disorders. The COVID-19 pandemic was specified as the traumatic exposure to record peritraumatic and posttraumatic symptoms associated with the range of stressors introduced by the COVID-19 pandemic. Those who scored $\geq 1.75$ out of 4 were considered symptomatic.

$\S$ Passive suicidal ideation was assessed using an item from the Columbia-Suicide Severity Rating Scale adapted to refer to the past 30 days: "At any time in the past 30 days, have you wished you were dead or wished you could go to sleep and not wake up?" Serious suicidal ideation was assessed using an item from the National Survey on Drug Use and Health adapted to refer to the past 30 days: "At any time in the past 30 days, did you seriously think about trying to kill yourself?"

" Weighted numbers and percentages might not sum to expected values because of rounding.

** Parents and unpaid caregivers of adults were self-identified. For this analysis, parents were defined as persons who had provided unpaid care to relatives or friends aged $<18$ years to help them take care of themselves at any time in the last 3 months. Unpaid caregivers of adults were defined as persons who had provided unpaid care to relatives or friends aged $\geq 18$ years to help them take care of themselves at any time in the last 3 months. Respondents answered questions about these two roles separately. Respondents were categorized as parents only, caregivers of adults only, parents-caregivers (persons in both roles), or nonparents/ noncaregivers. Whether adults in parenting roles were biologic or legal parents or guardians of the children for whom they were providing care is not known, nor is it known whether adults were legal dependents of their caregivers.

${ }^{+\dagger}$ Referent: nonparent/noncaregiver. Weighted multivariable logistic regression models were used to estimate aORs for each adverse mental health symptom, with survey wave, gender, age group, race/ethnicity, sexual orientation, disability status, education attainment, region, urbanicity, and employment (work hours per week and remote work percentage) as covariates. P values $<0.05$ were considered statistically significant. Models with all unpaid caregiver statuses included 10,017 respondents because persons who answered "prefer not to say" for sexual orientation or disability status and those who reported invalid zip codes were excluded. Models with unpaid caregivers of adults included 3,155 respondents; respondents were excluded for the same reasons.

$\S \S$ Referent: not providing care to an adult for this reason. This referent group includes all adults not providing care for the listed reason, including those who were nonparents/noncaregivers, parents only, and caregivers of adults who were providing care for different reasons. 
FIGURE. Factors* associated ${ }^{\dagger}$ with adverse mental health symptoms ${ }^{\S}$ among unpaid caregivers of adults and parents-caregivers ${ }^{\Uparrow}-$ The $^{*}$ COVID-19 Outbreak Public Evaluation Initiative, United States, December 2020 and February-March 2021

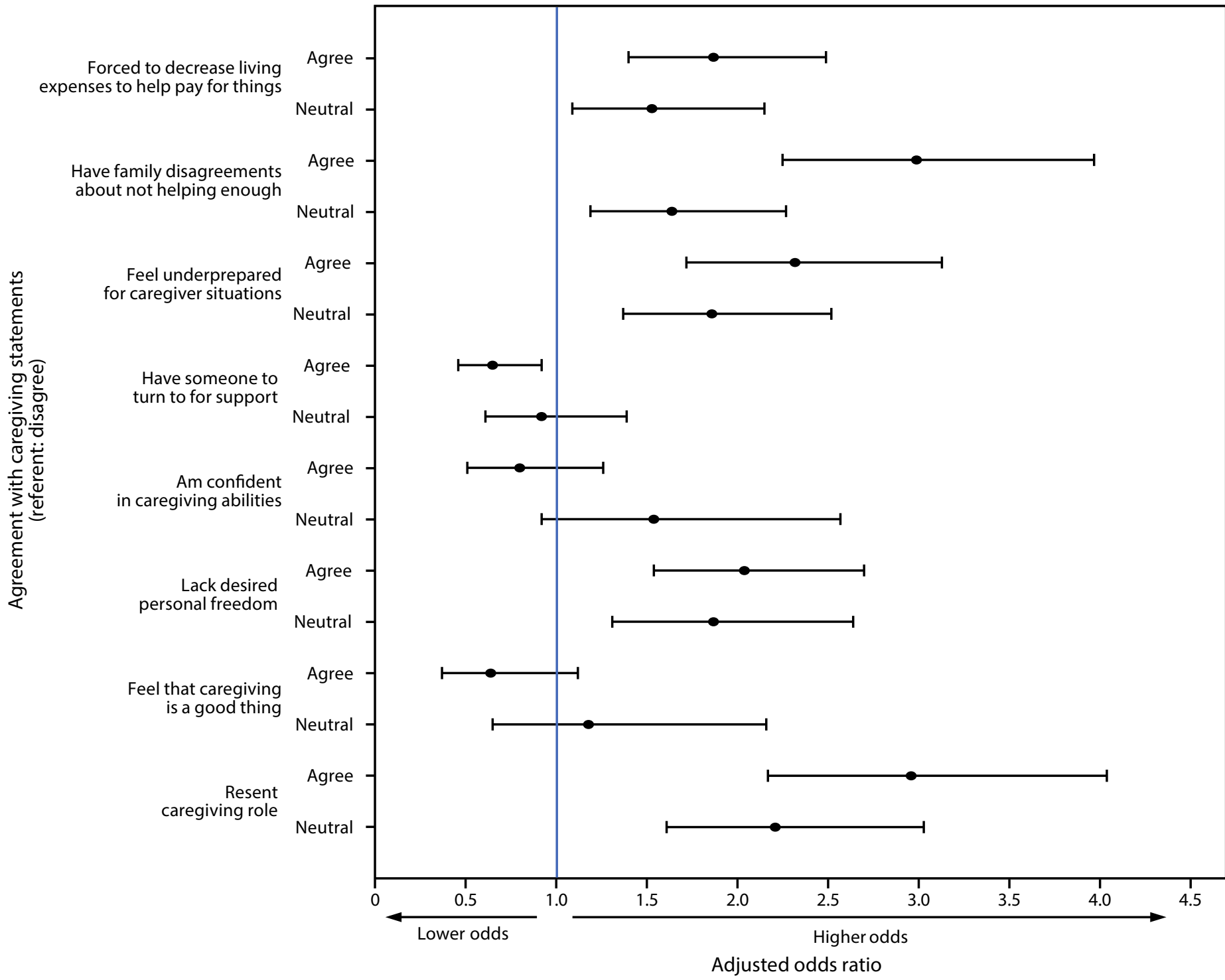

Abbreviations: ASD = acute stress disorder; CII = Caregiving Intensity Index; GAD-2 = two-item Generalized Anxiety Disorder scale; IES-6 = six-item Impact of Event scale; PHQ-2 = two-item Patient Health Questionnaire; PHQ-4 = four-item Patient Health Questionnaire; PTSD = posttraumatic stress disorder; TSRD = trauma- and stressor-related disorder. * Caregiving statements were taken from the ARCHANGELS short-form Cll, a copyrighted instrument available for use only with permission.

${ }^{\dagger}$ Adjusted odds ratios, with $95 \%$ confidence intervals indicated by error bars, were estimated using weighted multivariable logistic regression models. The primary model is adjusted for survey wave, gender, age group, race/ethnicity, sexual orientation, disability status, education attainment, region, urbanicity, and employment (including work hours per week and remote work percentage), parental status (i.e., whether caregivers were parents-caregivers), duration of caregiving, hours of caregiving per week, and person receiving care. Additional separate models were analyzed for each Cll item that was based on perceived levels of agreement with statements regarding caregivingrelated financial strain, family strife, preparedness, support, confidence, personal freedom, positive feelings, and resentment.

$\S$ The presence of one or more of the following was considered an adverse mental health symptom: anxiety symptoms, depression symptoms, COVID-19 TSRD symptoms, passive suicidal ideation, or having seriously considered suicide in the past 30 days. Symptoms of anxiety and depression were assessed via PHQ-4. Those who scored $\geq 3$ out of 6 on the GAD-2 and PHQ-2 subscales were considered symptomatic for these respective conditions. Disorders classified as TSRDs in the Diagnostic and Statistical Manual of Mental Disorders, Fifth Edition, include PTSD, ASD, and adjustment disorders, among others. Symptoms of a TSRD attributed to the COVID-19 pandemic were assessed via IES-6 to screen for overlapping symptoms of PTSD, ASD, and adjustment disorders. The COVID-19 pandemic was specified as the traumatic exposure to record peritraumatic and posttraumatic symptoms associated with the range of stressors introduced by the COVID-19 pandemic. Those who scored $\geq 1.75$ out of 4 were considered symptomatic. Passive suicidal ideation was assessed using an item from the Columbia-Suicide Severity Rating Scale adapted to refer to the past 30 days:"At any time in the past 30 days: have you wished you were dead or wished you could go to sleep and not wake up?" Serious suicidal ideation was assessed using an item from the National Survey on Drug Use and Health, adapted to refer to the past 30 days: "At any time in the past 30 days, did you seriously think about trying to kill yourself?"

" Parents and unpaid caregivers of adults were self-identified. Unpaid caregivers of adults were defined as persons who had provided unpaid care to relatives or friends aged $\geq 18$ years to help them take care of themselves at any time in the last 3 months. Parents were defined as persons who had provided unpaid care to relatives or friends aged $<18$ years to help them take care of themselves at any time in the last 3 months. Parents-caregivers had both roles. All unpaid caregivers of adults were included in this analysis, including caregivers of adults only (i.e., not parents) and parents-caregivers. 
suicide prevention ${ }^{\dagger \dagger \dagger \dagger \dagger}$ and mental health disaster support services $\$ \$ \$ \$ \$ \$, \mathbf{9 9 9 9 9 , * * * * * *}$ are needed for parents and caregivers.

During the pandemic, parents and caregivers have had worse mental health than adults without parenting and caregiving responsibilities (5). Managing mental health might be especially challenging for parents balancing employment and remote education; virtual instruction during the COVID-19 pandemic has presented risks for mental health both among children and parents ( 6 ). For caregivers of adults, these findings reinforce prepandemic data on poor mental health among caregivers (7). The results also support AmeriSpeak Omnibus survey findings that during the COVID-19 pandemic, caregivers had substantial concerns about their own mental health and the health and well-being of their care recipients, were worried about their finances, and needed respite from caregiving (8). Adverse mental health consequences for persons in both roles (i.e., parents-caregivers) support an urgent need to tailor public health efforts to this population. Together, these results suggest that parents and caregivers might benefit from tailored mental health services. For caregivers, and especially persons with dual responsibilities of parenting while also caring for adults, increasing access to, awareness of, and use of support groups and respite services ${ }^{\dagger \dagger+\dagger_{\dagger} \dagger}$ might help to alleviate the caregiving workload $\$ \$ \$ S \$(9)$.

The findings in this report are subject to at least six limitations. First, this study did not fully characterize parenting roles (e.g., age and number of children, whether children had chronic health conditions, and whether children were in virtual rather than in-person school). Whether the mental health of adults differs based on these factors could be explored. Second, diagnostic evaluations for anxiety and depression were not conducted; however, clinically validated instruments were used to measure symptoms of anxiety and depression. Third, responses might be subject to social desirability bias, particularly regarding negative feelings about caregiving roles, which might be underreported. Fourth, without prepandemic mental health data in this sample, whether adverse mental health symptoms were caused by or worsened by the pandemic is unknown. However, caregivers of adults had higher odds of new adverse mental or behavioral health symptoms during the pandemic than did noncaregivers

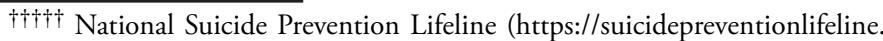
org/) or Lifeline Crisis Chat (https://suicidepreventionlifeline.org/chat/).

$\$ \mathbb{S} \$ \mathbb{S} \$$ Substance Abuse and Mental Health Services Administration National Helpline (also known as the Treatment Referral Routing Service) for persons and families facing mental disorders, substance use disorders, or both (https://www.samhsa.gov/find-help/national-helpline).

99999 Disaster Distress Helpline (https://www.samhsa.gov/disaster-preparedness).

****** Crisis Text Line (https://www.crisistextline.org/).

$+\dagger+\dagger^{\dagger \dagger}$ https://www.cdc.gov/aging/publications/features/caring-for-yourself.html; https://www.norc.org/PDFs/Maintaining\%20Physical\%20and\%20 Mental\%20Well/OACCaregiverOnePager.pdf

$\$ \$ \$ \$ \$ \$$ https://www.caregiving.org/resources/
}

\section{Summary}

What is already known about this topic?

Parents of children aged $<18$ years and unpaid caregivers of adults have had mental health challenges before and during the COVID-19 pandemic.

What is added by this report?

Among 10,444 U.S. adults surveyed during December 6-27, 2020, and February 16-March 8, 2021, parents, unpaid caregivers of adults, and parents-caregivers (persons in both roles) had significantly worse mental health than adults not in these roles, including five times the odds of any adverse mental health symptoms (parents-caregivers). Persons who had someone to rely on for support had lower odds of experiencing any adverse mental health symptoms.

What are the implications for public health practice?

Parents and unpaid caregivers of adults, and particularly those in both roles, might benefit from mental health support and services tailored to their roles.

(1). Fifth, the survey did not assess support systems for parents or caregivers (e.g., child care or support from family members), which could have affected the intensity of their caregiving roles. Finally, because the surveys were English-language only and quota sampling and survey weighting might not have eliminated inherent biases in Internet-based survey samples, 999999 this sample might not fully represent the U.S. population, particularly regarding English-language fluency and Internet access. This might partially account for the finding that more parents, caregivers, or parents-caregivers were male. However, previous studies have estimated that up to $47 \%$ of caregivers are male. Furthermore, the infrequency of assessments of both parental and caregiving roles makes comparing these estimates difficult. ${ }^{* * * * * * * *}$ The prevalence and trajectories of anxiety and depression symptoms were consistent with results from the Household Pulse Survey $\dagger^{\dagger \dagger \dagger \dagger \dagger \dagger}(10)$, and robust associations between parenting and caregiving roles and adverse mental health symptoms in the large, demographically diverse COPE Initiative sample merit additional research.

Caregivers, particularly persons with both parenting and adult caregiving responsibilities, will continue to face mental health challenges, and the need for caregivers is projected to increase as the U.S. population ages. ${ }^{\$ S \$ S S \$ S}$ Additional research can assess differences in coping and help-seeking behaviors among parents and caregivers to further guide tailored support and services to meet their needs during and after the COVID-19 pandemic.

\footnotetext{
999999 https://www.pewresearch.org/politics/methodology/collecting-surveydata/internet-surveys/

******* https://www.apa.org/pi/about/publications/caregivers/faq/statistics

††计计 https://www.cdc.gov/nchs/covid19/pulse/mental-health.htm

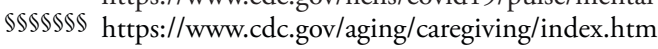




\section{Acknowledgments}

The COPE Initiative survey respondents; Mallory Colys, Sneha Baste, Daniel Chong, Rebecca Toll, Qualtrics, LLC; CDC Foundation, with funding from BNY Mellon; Hopelab, Inc.; Lisa C. McGuire, Rashon I. Lane, CDC; The Kinghorn Foundation; Australian-American Fulbright Commission.

Corresponding author: Elizabeth Rohan, Erohan@cdc.gov.

\begin{abstract}
${ }^{1}$ Turner Institute for Brain and Mental Health and School of Psychological Sciences, Monash University, Melbourne, Australia; ${ }^{2}$ Austin Health, Melbourne, Australia; ${ }^{3}$ Brigham \& Women's Hospital, Boston, Massachusetts; ${ }^{4} \mathrm{CDC}$ COVID-19 Response Team; ${ }^{5}$ Harvard Medical School, Boston, Massachusetts; ${ }^{6}$ ARCHANGELS, Boston, Massachusetts; ${ }^{7}$ WHOOP, Inc., Boston, Massachusetts; ${ }^{8}$ University of Melbourne, Melbourne, Australia.
\end{abstract}

All authors have completed and submitted the International Committee of Medical Journal Editors form for disclosure of potential conflicts of interest. Laura K. Barger reports institutional grants paid to Monash University from the CDC Foundation, with funding provided by BNY Mellon and from WHOOP, Inc., and consulting fees from AAA Foundation, CurAegis, University of Helsinki, Puget Sound Pilots, Boston Children's Hospital, and Charles A. Czeisler. Emily R. Capodilupo reports grant options in Rebel Health, Inc., doing business as ARCHANGELS (ARCHANGELS) as a member of the board of advisors and shares held in WHOOP, Inc., of which she is a paid employee. Mark É. Czeisler reports institutional grants paid to Monash University by the CDC Foundation, with funding provided by BNY Mellon, and WHOOP, Inc.; support from the Australian-American Fulbright Foundation, with funding provided by The Kinghorn Foundation; and consulting fees from Vanda Pharmaceuticals. Alexandra Drane reports employment by ARCHANGELS, which receives grant funding from the Ralph C. Wilson Junior Foundation in support of the development of testing and rollout of the Caregiver Intensity Platform; institutional licensing by ARCHANGELS; personal consulting fees and personal speaking fees from Prudential Financial Services; institutional consulting fees from ARCHANGELS on the topic of caregiving; membership on the RAND Health Advisory Board, the United States of Care Entrepreneurs Council, the Division of Sleep Medicine, Harvard Medical School Executive Council, the Massachusetts Technology Collaborative Executive Committee for the Board of Directors, the Coalition to Transform Advanced Care Board of Directors, the EndWell Advisory Board, the Beth Israel Deaconess Medical Center Trustee Advisory Board as vice chair, the MassChallenge Health Tech Advisory Board, the Boston Children's Hospital Innovation and Digital Health Accelerator Board of Advisors, the Edenbridge Health Advisory Board, the Health Evolution Leadership Committee, and the Rosalynn Carter Institute for Caregivers Leadership Council; and an equity interest in ARCHANGELS, a minority interest in HPT Development/Drane Associates, and investments in WHOOP, Inc., Avanlee, and Town Hall Ventures. Mark E. Howard reports institutional grants paid to Monash University from the CDC Foundation, with funding provided by BNY Mellon, and from
WHOOP, Inc., Shantha M.W. Rajaratnam reports institutional grants paid to Monash University from the CDC Foundation, with funding provided by BNY Mellon, and from WHOOP, Inc., and the Cooperative Research Centre for Alertness, Safety and Productivity and institutional consulting fees paid to Monash University from Teva Pharma Australia, Circadian Therapeutics, BHP Billiton, and Herbert Smith Freehills. Rebecca Robbins reports institutional grants paid to Monash University from the CDC Foundation, with funding provided by BNY Mellon, and from WHOOP, Inc., and consulting fees from Denihan Hospitality, Rituals Cosmetics, SleepCycle, Dagmejan, and byNacht. Matthew D. Weaver reports institutional grants paid to Monash University from the CDC Foundation, with funding provided by BNY Mellon and from WHOOP, Inc.; institutional grants from the Brigham and Women's Physician's Organization, the Brigham Research Institute, the National Institute for Occupational Safety and Health, and the National Heart, Lung, and Blood Institute; and consulting fees from the National Sleep Foundation and the University of Pittsburgh. Joshua F. Wiley reports institutional grants paid to Monash University from the CDC Foundation, with funding provided by BNY Mellon, and from WHOOP, Inc., and a grant from the National Health and Medical Research Council Fellowship. Sarah S. Winnay reports employment by ARCHANGELS, which receives grant funding from the Ralph C. Wilson, Jr. Foundation, and institutional license by ARCHANGELS for ARCHANGELS Caregiver Intensity Index, and consulting and speaking fees for services provided by ARCHANGELS and equity holding in ARCHANGELS. Charles A. Czeisler reports institutional grants paid to Monash University from the CDC Foundation, with funding provided by BNY Mellon, and from WHOOP, Inc.; grants and contracts from Delta Airlines, Jazz Pharmaceuticals, PLC, INC., Philips Respironics, Inc., Puget Sound Pilots, Regeneron Pharmaceuticals and Sanofi SA, ResMed, Teva Pharmaceuticals Industries, Ltd., and Vanda Pharmaceuticals; royalty payments from Philips Respironics, Inc; consulting fees from Physician's Seal, State of Washington Board of Pilotage Commissioners, and Vanda Pharmaceuticals; honoraria/payment from Teva Pharma Australia, Tencent Holdings, Ltd., and the National Sleep Foundation; Advisory Board membership for AARP, Institute of Digital Media and Child Development, the Klarman Family Foundation, and the UK Biotechnology and Biological Sciences Research Council; equity interest in Vanda Pharmaceuticals; an institutional educational gift to Brigham and Women's Hospital from Alexandra Drane, Johnson \& Johnson, and Harmony Biosciences, LLC; and an endowed professorship (incumbent) provided to Harvard Medical School by Cephalon, Inc. Elise R. Facer-Childs reports institutional grants paid to Monash University from the CDC Foundation, with funding provided by BNY Mellon, and from WHOOP, Inc., and a Science and Industry Endowment Fund Ross Metcalf STEM+ Business Fellowship administered by the Commonwealth Scientific and Industrial Research Organisation. No other potential conflicts of interest were disclosed. 


\section{References}

1. Czeisler MÉ, Lane RI, Petrosky E, et al. Mental health, substance use, and suicidal ideation during the COVID-19 pandemic-United States, June 24-30, 2020. MMWR Morb Mortal Wkly Rep 2020;69:1049-57. PMID:32790653 https://doi.org/10.15585/mmwr.mm6932a1

2. Patrick SW, Henkhaus LE, Zickafoose JS, et al. Well-being of parents and children during the COVID-19 pandemic: a national survey. Pediatrics 2020;146:e2020016824. PMID:32709738 https://doi.org/10.1542/ peds.2020-016824

3. Sherifali D, Ali MU, Ploeg J, et al. Impact of Internet-based interventions on caregiver mental health: systematic review and meta-analysis. J Med Internet Res 2018;20:e10668. PMID:29970358 https://doi. org/10.2196/10668

4. Parker LJ, Gaugler JE, Samus Q, Gitlin LN. Adult day service use decreases likelihood of a missed physician's appointment among dementia caregivers. J Am Geriatr Soc 2019;67:1467-71. PMID:31219175 https://doi. org/10.1111/jgs. 15995

5. Czeisler MÉ, Lane RI, Wiley JF, Czeisler CA, Howard ME, Rajaratnam SMW. Follow-up survey of US adult reports of mental health, substance use, and suicidal ideation during the COVID-19 pandemic, September 2020. JAMA Netw Open 2021;4:e2037665. PMID:33606030 https:// doi.org/10.1001/jamanetworkopen.2020.37665
6. Verlenden JV, Pampati S, Rasberry CN, et al. Association of children's mode of school instruction with child and parent experiences and wellbeing during the COVID-19 pandemic-COVID Experiences Survey, United States, October 8-November 13, 2020. MMWR Morb Mortal Wkly Rep 2021;70:369-76. PMID:33735164 https://doi.org/10.15585/ mmwr.mm7011a1

7. Schulz R, Sherwood PR. Physical and mental health effects of family caregiving. Am J Nurs 2008;108(Suppl):23-7. PMID:18797217 https:// doi.org/10.1097/01.NAJ.0000336406.45248.4c

8. NORC at the University of Chicago. Needs assessment and environmental scan report: maintaining physical and mental well-being of older adults and their caregivers during public health emergencies. Bethesda, MD: NORC at the University of Chicago; 2021. https://www. norc.org/PDFs/Maintaining\%20Physical\%20and\%20Mental\%20Well/ ESandNAReportNarrative.pdf

9. Worrall H, Schweizer R, Marks E, Yuan L, Lloyd C, Ramjan R. The effectiveness of support groups: a literature review. Mental Health and Social Inclusion 2018;22:85-93. https://doi.org/10.1108/ MHSI-12-2017-0055

10. Vahratian A, Blumberg SJ, Terlizzi EP, Schiller JS. Symptoms of anxiety or depressive disorder and use of mental health care among adults during the COVID-19 pandemic - United States, August 2020-February 2021. MMWR Morb Mortal Wkly Rep 2021;70:490-4. PMID:33793459 https://doi.org/10.15585/mmwr.mm7013e2 